University of Massachusetts Amherst

ScholarWorks@UMass Amherst

Physics Department Faculty Publication Series

Physics

2008

\title{
Bold diagrammatic Monte Carlo: A generic sign- problem tolerant technique for polaron models and possibly interacting many-body problems
}

N Prokof'ev

University of Massachusetts - Amherst, prokofev@physics.umass.edu

B Svistunov

University of Massachusetts - Amherst, svistunov@physics.umass.edu

Follow this and additional works at: https://scholarworks.umass.edu/physics_faculty_pubs

Part of the Physics Commons

\section{Recommended Citation}

Prokof'ev, N and Svistunov, B, "Bold diagrammatic Monte Carlo: A generic sign-problem tolerant technique for polaron models and possibly interacting many-body problems" (2008). Physics Review B. 1128.

Retrieved from https://scholarworks.umass.edu/physics_faculty_pubs/1128 


\title{
Bold diagrammatic Monte Carlo: A generic technique for polaron (and many-body?) problems
}

\author{
N.V. Prokof'ev ${ }^{1,2,3}$ and B.V. Svistunov ${ }^{1,3}$ \\ ${ }^{1}$ Department of Physics, University of Massachusetts, Amherst, MA 01003, USA \\ ${ }^{2}$ Theoretische Physik, ETH Zürich, CH-8093 Zürich, Switzerland \\ ${ }^{3}$ Russian Research Center "Kurchatov Institute", 123182 Moscow, Russia
}

\begin{abstract}
We develop a Monte Carlo scheme for sampling series of Feynman diagrams for the proper selfenergy which are self-consistently expressed in terms of renormalized particle propagators. This approach is used to solve the problem of a single spin-down fermion resonantly interacting with the Fermi gas of spin-up particles. Though the original series based on bare propagators are signalternating and divergent one can still determine the answer behind them by using two strategies (separately or together): (i) using proper series re-summation techniques, and (ii) introducing renormalized propagators which are defined in terms of the simulated proper self-energy, i.e. making the entire scheme self-consistent. Our solution is important for understanding the phase diagram and properties of the BCS-BEC crossover in the strongly imbalanced regime. On the technical side, we develop a generic sign-problem tolerant method for exact numerical solution of polaron-type models, and, possibly, of the interacting many-body Hamiltonians.
\end{abstract}

PACS numbers: 05.30.Fk, 05.10.Ln, 02.70.Ss

\section{INTRODUCTION}

Modern science has radically changed our view of the physical vacuum. Instead of naïve "empty space" we have to deal with a complex groundstate of an interacting system, and, strictly speaking, there is no fundamental difference between the outer space, helium, or any other condensed matter system. With this point of view comes understanding that the notion of a "bare" particle is somewhat abstract since its coupling to vacuum fluctuations, or environment, may strongly (sometimes radically) change particle properties at energies addressed by the experimental probes. The polaron problem [1] is by now canonical across all of physics with the same questions about particle energy, effective mass, residue, etc., being asked for different types of particles, environments and coupling between them [2]. In a broader context, particles are not necessarily external objects unrelated to a given vacuum, but quasiparticle excitations of the very same ground state. Thus, the solution of the polaron problem paves the way to the effective low energy theory of a given system, and, to large extent, determines basic properties of all condensed matter systems at low temperature.

At the moment, there is no generic analytic or numeric technique to study quasiparticle properties for arbitrary strongly interacting system. Analytic solutions are typically (with few exceptions, see, e.g., [3]) based on perturbative corrections to certain limiting cases [1, 2, , 4, 5, 6] or variational treatment [7]. Several numeric schemes were suggested in the past, but all of them have limitations either in the system size, system dimension, interaction or environment type. Exact diagonalization and variational methods in the low-energy subspace [8, 9] are mostly restricted to one-dimensional models with shortrange interactions. The continuous time path integral formulation [10] works for the lattice model with linear coupling between the particle and bosonic environment, but it can not be generalized to fermionic environment, sign-alternating coupling (as in the $t$ - $J$ model [11]), nor is it suitable for continuous-space models.

In this article (which follows a short communication 12]), we develop a Monte Carlo technique which simulates series of Feynman diagrams for the proper selfenergy. The diagrammatic Monte Carlo (Diag-MC) technique was used previously to study electron-phonon polarons [13, 14]. The essence of Diag-MC is in interpreting the sum of all Feynman diagrams as an ensemble averaging procedure over the corresponding configuration space. It was considered essential for the method to work that the series of diagrams for the Green's function be convergent and sign-positive. Though the configuration space of diagrams for polarons in Fermi systems is more complex, similar methods of generating and sampling the corresponding configuration space can be used. The crucial difference is that in the Fermi system we have to deal with the sign-alternating and divergent (at least for strong coupling) series. Under these conditions a direct summation of all relevant Feynman diagrams for the Green's functions is not possible, and one has to develop additional tools for (i) reducing the number of diagrams by calculating self-energies rather than Green's functions, (ii) employing the "bold-line" trick in the form of the Dyson equation which allows self-consistent summation of infinite geometric series and further reduces the number of self-energy diagrams, and, if necessary, (iii) extrapolating Diag-MC results to the infinite diagram order for a divergent series. At the moment we do not see any obvious limitations of the new method since even divergent sign alternating series can be dealt with to obtain reliable results. We believe that our findings are important in a much broader context since the Diag-MC approach to the many-body problem has essentially the same structure.

As a practical application of the method we consider a 
particle coupled to the ideal Fermi sea via a short-range potential with divergent $s$-wave scattering length. This problem was recognized as the key one for understanding the phase diagram of the population imbalanced resonant Fermi gas [15, 16]. In particular, to construct the energy functional describing dilute solutions of minority (spin down) fermions resonantly coupled to the majority (spin up) fermions one has to know precisely the quasiparticle parameters of spin-down fermions since they determine the coefficients in the energy expansion in the spin-down concentration $x_{\downarrow}$ : the linear term is controlled by the polaron energy, and the $x_{\downarrow}^{5 / 3}$ term is determined by the polaron mass [16].

The general Hamiltonian we deal with in this article can be written as

$$
H=H_{F}-\Delta_{R} / 2 m+\int d \boldsymbol{r} V(\boldsymbol{r}-\boldsymbol{R}) n(\boldsymbol{r}),
$$

where $H_{F}$ is the Hamiltonian of the ideal spin-up Fermi gas with density $n$ and Fermi momentum $k_{F}, \boldsymbol{R}$ is the particle coordinate, and $V(\boldsymbol{r}-\boldsymbol{R})$ is the interaction potential of finite range $r_{0}$ between the particle and the spin-up Fermi gas. In what follows we refer to (1) as the Fermi-polaron problem. The specifics of the BCS-BEC crossover physics in the strongly imbalanced regime is two-fold: one is that the particle and the Fermi gas have the same bare mass $m$ (in what follows we will use units such that $m=1 / 2$ and $k_{F}=1$ ), and the other is that one has to take explicitly the so-called zero-range resonant limit when $r_{0} \rightarrow 0$, but the $s$-scattering length $a$ remains finite, i.e. $k_{F} a$ remains fixed for $k_{F} r_{0} \rightarrow 0$. In this limit, the nature of the interaction potential is irrelevant, and the same results will be obtained e.g. for the neutron matter and Cesium atoms. We note, however, that the method we develop for the numeric solution of the resonant Fermi-polaron problem is absolutely general and can be used for an arbitrary model described by Eq. (1).

It turns out that the structure of the phase diagram is very sensitive to polaron parameters. If the state with a dilute gas of spin-down fermions is stable at all values of $k_{F} a$ then the solution of the single-particle problem would define the phase diagram in the vicinity of the multicritical point discussed recently by Sachdev and Yang [17], where four different phases meet. At this point the spin-down fermion forms a bound state with a spinup fermion thus becoming a spin-zero composite boson ("molecule"); i.e. quasi-particles radically change their statistics. The multicritical point, however, may be thermodynamically unstable if the effective scattering length between the composite bosons and spin-up electrons is large enough, and the analysis of Refs. [18, 19] based on the fixed-node Monte Carlo simulations finds evidence in favor of this scenario. Phase separation was also found in calculations based on mean-field and narrow-resonance approaches (both at finite and zero temperature) see e.g. Refs. 20, 21, 22, 23, 24, 25], though with strong quantitative deviations from results based on the fixed-node
Monte Carlo simulations [19]. On the experimental side, MIT experiments [26] are in good agreement with the predictions made in Ref. [19], while Rice experiments 27] are not. The origin of discrepancy between the two experiments is not understood. Our results for polaron energies are in excellent agreement with Ref. [19].

It is worth noting that the model (1) (in general, the particle mass is different from that of the Fermi gas) is also known as the Anderson orthogonality problem with recoil [28, 29]. It can be also considered as a specific example of a particle coupled to the Ohmic dissipative bath (see monograph [30] for numerous other examples and connections to realistic systems).

The paper is organized as follows. In Sec. [II we discuss the configuration space of Feynman diagrams for self-energy in momentum-imaginary-time representation (both in the particle and molecule channels), and explain how polaron parameters can be obtained in this representation. In Sec. III we describe a Monte Carlo algorithm for generating and sampling the corresponding diagrammatic space. A small technical Section IV deals with numerically evaluating the effective $T$-matrix by bold diagrammatic Monte Carlo. We present and discuss results in Sec.V. In particular, we show that one can use re-summation techniques for divergent series of diagrams based on bare propagators to determine the final answer. In Sec. VI we further advance the algorithm by employing bold-line approach in which the entire scheme is self-consistently based on renormalized ("bold-line") propagators. We present our conclusions and perspectives for future work in Sec. VII.

\section{CONFIGURATION SPACE OF SELF-ENERGY DIAGRAMS}

As mentioned above, when coupling between spindown and spin-up fermions is strong enough they from a composite boson, or molecule state. In what follows, we will be using the term "polaron" in a narrow sense, i.e. only for the unbound fermionic spin-down excitation. For the composite boson we will be using the term "molecule". Since our goal is to calculate particle properties for arbitrary coupling strength we have to consider one- and two-particle channels on equal footing. In the rest of this section we render standard diagrammatic rules for irreducible self-energy diagrams in both channels, with an emphasis on specifics of working in the imaginary-time representation.

\section{A. Polaron channel}

We start from the definition of the single-particle Green's function (see e.g. [31])

$$
G(\tau, \mathbf{r})=-\left\langle T_{\tau} \psi(\tau, \mathbf{r}) \bar{\psi}(0)\right\rangle,
$$


and its frequency-momentum representation

$$
G(\xi, \mathbf{p})=\int \mathrm{e}^{i(\xi \tau-\mathbf{p} \cdot \mathbf{r})} G(\tau, \mathbf{r}) d \mathbf{r} d \tau .
$$

Here $\psi(\tau, \mathbf{r})$ is the fermion annihilation operator at the space-time point $(\tau, \mathbf{r})$. For the ideal spin-up Fermi gas at $T=0$ we have

$$
G_{\uparrow}(\xi, \mathbf{p})=\frac{1}{i \xi-p^{2} / 2 m+\epsilon_{F}} .
$$

The vacuum Green's function for the spin-down polaron is

$$
G_{\downarrow}^{(0)}(\tau, \mathbf{p}, \mu)=-\theta(\tau) \mathrm{e}^{-\left(p^{2} / 2 m-\mu\right) \tau},
$$

where $\theta$ is the step function, and $\mu$ is a free parameter. From Dyson's equation for the polaron, see Fig. 1, one finds

$$
G_{\downarrow}(\xi, \mathbf{p}, \mu)=\frac{1}{i \xi-p^{2} / 2 m+\mu-\Sigma(\xi, \mathbf{p}, \mu)},
$$

where self-energy $\Sigma$ is given by the sum of all irreducible diagrams (i.e. diagrams which can not be made disconnected but cutting through the $G_{\downarrow}^{(0)}$ line) taken with the negative sign. Taking into account that in the $\tau$ representation both $G \downarrow$ and $\Sigma$ depend on $\mu$ only through exponential factors $\exp (\mu \tau)$, one obtains $G_{\downarrow}(\xi, \mathbf{p}, \mu) \equiv$ $G_{\downarrow}(\xi-i \mu, \mathbf{p})$ and $\Sigma(\xi, \mathbf{p}, \mu) \equiv \Sigma(\xi-i \mu, \mathbf{p})$.

If polaron is a well-defined quasi-particle, then its energy $E(\mathbf{p})$ and residue $Z(\mathbf{p})$ can be extracted from the asymptotic decay

$$
G_{\downarrow}(\tau, \mathbf{p}, \mu) \rightarrow-Z \mathrm{e}^{-(E-\mu) \tau}, \quad \tau \rightarrow \infty .
$$

This asymptotic behavior immediately implies that the function $G_{\downarrow}(\xi-i \mu, \mathbf{p})$ has a pole singularity

$$
G_{\downarrow}(\xi-i \mu, \mathbf{p})=\frac{Z(\mathbf{p})}{i \xi+\mu-E(\mathbf{p})}+\text { regular part } .
$$

Now setting $\mu=E(\mathbf{p})$ in (8) and comparing the result to (6), we conclude that

$$
i \xi / Z=i \xi-p^{2} / 2 m+E-\Sigma(0, \mathbf{p}, E)+i \xi A(\mathbf{p}, E),
$$

where (we take into account that $\partial \Sigma / \partial \xi=i \partial \Sigma / \partial \mu$ )

$$
A(\mathbf{p}, E)=-\left.\frac{\partial \Sigma(0, \mathbf{p}, \mu)}{\partial \mu}\right|_{\mu=E} .
$$

This yields two important relations (see also [31]):

$$
E=p^{2} / 2 m+\Sigma(0, \mathbf{p}, E),
$$

and

$$
Z=\frac{1}{1+A(\mathbf{p}, E)}
$$

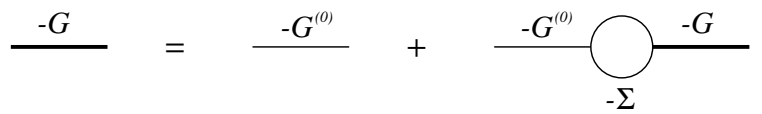

FIG. 1: Dyson equation for the single-particle Green's function.

Equation (11) allows one to solve for $E$ provided $\Sigma(\tau, \mathbf{p}, \mu)$ is known. All we have to do is to calculate the the zero-frequency value of $\Sigma$ for $\mu=E$

$$
E=p^{2} / 2 m+\int_{0}^{\infty} \Sigma(\tau, \mathbf{p}, \mu) \mathrm{e}^{(E-\mu) \tau} d \tau .
$$

After $E$ is found, the residue is obtained from Eq. (12) using

$$
A(\mathbf{p}, E)=-\int_{0}^{\infty} \tau \Sigma(\tau, \mathbf{p}, \mu) \mathrm{e}^{(E-\mu) \tau} d \tau .
$$

Note also that the dependence on $\mu$ drops out from both (13) and (14).

A comment is in order here. Strictly speaking, polaron and molecule poles exist only for $\mathbf{p}=0$ because the fermionic bath they couple to is gapless. However, the spectrum $E(\mathbf{p})$ is well-defined in the limit $p \rightarrow 0$, as the decay width vanishes faster than $[E(\mathbf{p})-E(0)]$. To have stable quasi-particles, one can use a trick of introducing a gap $\Delta$ in the environment spectrum, e.g. by redefining the dispersion relation for spin-up fermions: $\varepsilon_{\mathbf{k}} \rightarrow \max \left(\varepsilon_{\mathbf{k}}, \Delta\right)$. In the $p \rightarrow 0$ limit, the systematic error vanishes faster than $[E(\mathbf{p})-E(0)]$, provided $\Delta \sim[E(\mathbf{p})-E(0)]$. It should be also possible to work with $\Delta$ 's essentially larger than $[E(\mathbf{p})-E(0)]$ and extrapolate to $\Delta \rightarrow 0$. In particular, such an extrapolation is possible (and is implicitly implied) at the analytical level in the relation for the effective mass, which we consider below.

One way to determine the effective mass is to calculate the quasi-particle energy as a function of momentum for a set of small but finite values of $p$ and fit it with the parabola. It is, however, possible to skip this procedure and to write a direct estimator for the effective mass in terms of the calculated self-energy. Acting with the operator $\nabla_{\mathbf{P}}^{2}$ on both sides of Eq. (11) and taking the limit $p \rightarrow 0$ we get

$$
\begin{gathered}
\frac{1+A_{0}}{m_{*}}=\frac{1}{m}+B_{0}, \\
B_{0}=\left.\frac{1}{3} \int_{0}^{\infty} d \tau \mathrm{e}^{\left(E_{0}-\mu\right) \tau}\left[\nabla_{\mathbf{P}}^{2} \Sigma(\tau, \mathbf{p}, \mu)\right]\right|_{p=0},
\end{gathered}
$$

where $A_{0} \equiv A(\mathbf{p}=0)$ and $E_{0} \equiv E(\mathbf{p}=0)$.

\section{B. Molecule channel}

In this case we start with the two-particle propagator

$$
K(\tau, \mathbf{p})=-\left\langle\mathrm{T}_{\tau} \Phi_{\mathbf{p}}(\tau) \Phi_{\mathbf{p}}^{\dagger}(0)\right\rangle,
$$




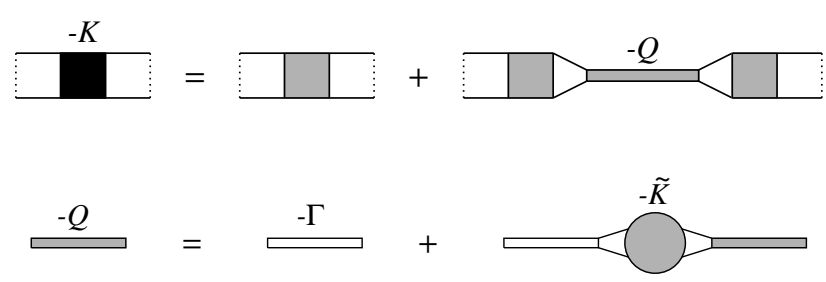

FIG. 2: Defining functions $Q$ and $\tilde{K}$ in the two-particle channel.

where

$$
\Phi_{\mathbf{p}}=\int \frac{d \mathbf{q}}{2 \pi} \varphi_{\mathbf{q}} \psi_{\uparrow}(\mathbf{p}-\mathbf{q}) \psi_{\downarrow}(\mathbf{q})
$$

and $\varphi_{\mathbf{q}}$ is the pair wavefunction (in momentum representation) that localizes the relative distance between two particles. If there is a bound state (molecule), then

$$
K(\tau, \mathbf{p}, \mu) \rightarrow-Z_{\mathrm{mol}} \mathrm{e}^{-\left(E_{\mathrm{mol}}-\mu\right) \tau}, \quad \tau \rightarrow \infty,
$$

and the pair propagator in the frequency representation has a pole:

$$
K(\xi-i \mu, \mathbf{p})=\frac{Z_{\mathrm{mol}}(\mathbf{p})}{i \xi+\mu-E_{\mathrm{mol}}(\mathbf{p})}+\text { regular part }
$$

Now we introduce yet another function, that features the same molecular pole, but has a simpler diagrammatic structure. The specifics of the resonant zero-range limit is that the sum of all ladder diagrams for the interaction potential $V(r)$ has to be considered as a separate diagrammatic element. We denote this sum by $\Gamma(\tau, p)$ and consider it as a "bare pair propagator". Of course, the same approach can be taken in a general case to replace the bare interaction potential with the scattering $T$ matrix, but in the zero-range limit we really do not have any other alternative for the ultra-violet-divergence-free formulation. The sum of ladder diagrams takes the ultraviolet physics into account exactly and allows to express $\Gamma(\tau, p)$ in terms of the $s$-scattering length $a$. The ladder structure of diagrams absorbed in $\Gamma(\tau, p)$ also explains why we treat it as a "pair propagator" (we will depict it with a double-line, see Fig 2). The exact expression for $\Gamma$ is readily obtained from the geometrical series (in frequency domain)

$$
-\Gamma=-U+(-U)^{2} \Pi+\ldots,
$$

where the polarization operator is defined by

$$
\Pi(\eta, p)=\int_{q>k_{F}} \frac{d \mathbf{q} /(2 \pi)^{3}}{q^{2} / 2 m+(\mathbf{p}-\mathbf{q})^{2} / 2 m-\eta},
$$

and $\eta=\omega+\varepsilon_{F}+\mu$. Using suitable ultra-violet regularization, one can cast the same expression in the universal form which depends only on the $s$-scattering length:

$$
\Gamma^{-1}(\eta, p)=\frac{m}{4 \pi a}-\frac{m}{8 \pi} \sqrt{p^{2}-4 m \eta}-\bar{\Pi}(\eta, p),
$$

$$
\bar{\Pi}(\eta, p)=\int_{q \leq k_{F}} \frac{d \mathbf{q} /(2 \pi)^{3}}{q^{2} / 2 m+(\mathbf{p}-\mathbf{q})^{2} / 2 m-\eta} .
$$

For finite density of spin-up fermions converting Eq. (23) to the imaginary time domain has to be done numerically. One possibility is to use the inverse Laplace transform. We employed the bold diagrammatic Monte Carlo technology 32] to achieve this goal and further details are provided in Sec. IV. The two-dimensional function $\Gamma(\tau, p)$ is tabulated prior to the polaron simulation.

In Fig. 2 we define function $Q$ that can be viewed as a renormalized pair propagator related to $\Gamma$ by the Dyson equation. In the upper panel, we show the diagrammatic structure for $K$, which includes dotted lines representing external functions $\varphi_{\mathbf{q}}$, grey squares representing sums of all $\Gamma$-irreducible diagrams, and the renormalized pair propagator $Q$. By $\Gamma$-irreducible diagrams we understand diagrams which can not be made disconnected by cutting them through a single $\Gamma$-line. All $\Gamma$-reducible diagrams are absorbed in the $Q$ function which is shown in the lower panel in Fig. 2. The grey circle has nearly the same structure as the grey square (the zeroth order term is present in the crossed square, but not in the crossed circle): since $\Gamma$ is defined as the sum of ladder diagrams, all terms which include ladder-type structures based on free one-particle propagators have to be excluded from $Q$ and $K$. With the replacements $G_{\downarrow} \rightarrow Q, G_{\downarrow}^{(0)} \rightarrow \Gamma$, and $\Sigma \rightarrow \tilde{K}$ we find an exact analogy between the one- and two-particle propagators.

The analogy can be carried out further by noting that the structure of diagrams in Fig. 2 implies that $Q$ has the same pole as $K$, while the rest of the functions simply change the value of the quasi-particle residue. Thus, if molecule is a well-defined excitation we expect that

$$
Q(\xi-i \mu, \mathbf{p})=\frac{\tilde{Z}_{\mathrm{mol}}(\mathbf{p})}{i \xi+\mu-E_{\mathrm{mol}}(\mathbf{p})}+\text { regular part } .
$$

This explains why introducing the function $Q$ is convenient: now Eqs. (12)-(16) are immediately generalized to the molecule case (up to replacements mentioned above).

\section{WORM ALGORITHM FOR FEYNMAN DIAGRAMS}

In this Section we describe how the configuration space of Feynman diagrams for $\Sigma$ and $\tilde{K}$ is parameterized and updated using Diag-MC rules. Our algorithm is designed to treat polaron and molecule channels on equal footing. We achieve this goal by introducing auxiliary diagrams which contain two "loose" ends called "worms" - this was proven to be an efficient strategy for reducing the MC autocorrelation time when simulations are performed in the configuration space with complex topology 33, 34. 


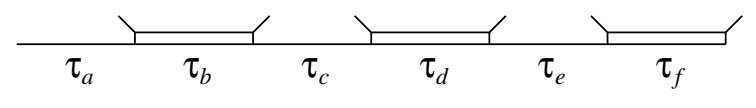

FIG. 3: The backbone of the cyclic diagram.

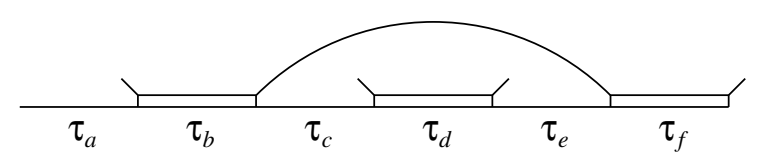

FIG. 4: Forward connection. The arc represents $-G_{\uparrow}(\tau=$ $\left.\tau_{c}+\tau_{d}+\tau_{e}\right)$.

\section{A. Cyclic diagrams}

We start with the introduction of cyclic diagrams. Though we work in the imaginary time representation at $T=0$ when $\tau \in[0, \infty)$, it is still convenient not to specify the time origin and to consider diagrams on the imaginary time circle. The backbone of each cyclic diagram is a pre-diagram illustrated in Fig. 3. It consists of a cyclic chain of the structure $G_{\downarrow}^{(0)}\left(\tau_{a}\right) \Gamma\left(\tau_{b}\right) G_{\downarrow}^{(0)}\left(\tau_{c}\right) \Gamma\left(\tau_{d}\right) G_{\downarrow}^{(0)}\left(\tau_{e}\right) \Gamma\left(\tau_{f}\right) \ldots$ (all the times are positive). We do not explicitly show "directions" of the propagators, since these are unambiguously fixed by the global direction of all the backbone lines, which we select - without loss of generality - to be from right to left. With this convention, the left (free) spinup end of any $\Gamma$-line is outgoing, while the right end is incoming. A physical diagram is obtained by pairwise replacing free spin-up ends with propagators $G_{\uparrow}$. There are two ways to connect incoming and outgoing lines: (i) forward, i.e., in the direction of the backbone propagators, and (ii) backward (opposite to forward). Forward (backward) connections result in propagators $G_{\uparrow}$ with positive (negative) times, see Figs. 4and 5 for illustrations. They represent particle (hole) excitations in the fermionic environment. It is important to emphasize that in cyclic diagrams the only time-variables are the positive timelengths of $G_{\downarrow}^{(0)}$ 's and $\Gamma$ 's. There is no absolute time, and, correspondingly, all moments in time are equivalent.

\section{B. Worms}

To have a MC scheme which simulates diagrams in one- and two-particle channels on equal footing we extend the space of physical diagrams by allowing diagrams with two special end-points, or "worms". They will be denoted $\mathcal{I}$ and $\mathcal{M}$ and stand for the unconnected incoming (outgoing) spin-up ends, see Fig. [6 for an illustration. Correspondingly, the entire updating scheme is based on

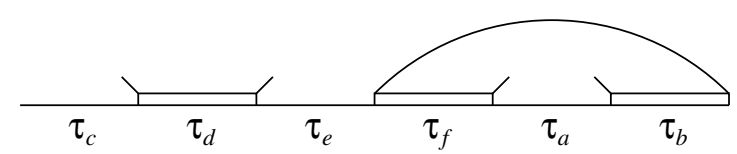

FIG. 5: Backward connection. The pair of $\Gamma$-ends being connected is precisely the same as in Fig. 4 but the direction is opposite, and the arc represents $-G_{\uparrow}\left(\tau=-\tau_{f}-\tau_{a}-\tau_{b}\right)$.

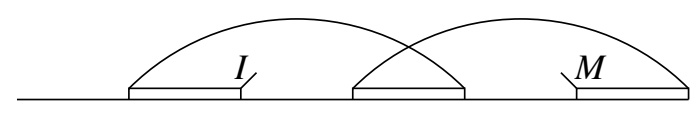

FIG. 6: A diagram with two worms, $\mathcal{I}$ and $\mathcal{M}$.

manipulations with $\mathcal{I}$ and $\mathcal{M}$. As it will become clear soon, of special importance for normalization of MC results is the first-order diagram with the worm, see Fig. 7 . Its weight consists of just two factors, $G_{\downarrow}^{(0)}\left(\tau_{a}\right)$ and $\Gamma\left(\tau_{b}\right)$.

\section{Parametrization of diagrams}

Apart from the diagram order and its topology, we select time intervals of $\Gamma$ 's and $G_{\downarrow}^{(0)}$ 's, and momenta of the spin-up propagators as independent variables. The momenta of $\Gamma$ 's and $G_{\downarrow}^{(0)}$ 's are then unambiguously defined by the momentum conservation law, while the time interval of a spin-up propagator is obtained by summing up the time intervals of $\Gamma$ 's and $G_{\downarrow}^{(0)}$ 's covered by this propagator. Technically, we find it convenient to work explicitly in the particle-hole representation for the spin-up propagators when backward spin-up propagator is understood as a forward hole propagator with the opposite momentum. This is achieved by introducing a non-negative function,

$$
\tilde{G}(\tau, \mathbf{p})=\left\{\begin{array}{l}
-G_{\uparrow}(\tau, \mathbf{p}), \quad p \geq p_{F}, \\
G_{\uparrow}(-\tau,-\mathbf{p}), \quad p<p_{F}
\end{array}\right.
$$

which is assigned to all spin-up lines (the global fermionic sign of the diagram is defined separately, by standard diagrammatic rules). All momenta assigned to the spinup lines are understood as momenta of the corresponding $\tilde{G}$-propagators; i.e. they are either momenta of particles (for forward propagators they are non-zero only for $p \geq$ $p_{F}$ ), or momenta of holes (for backward propagators they are non-zero only for $p<p_{F}$ ). An explicit formula for $\tilde{G}$ (subject to the above conditions) is

$$
\tilde{G}(\tau, \mathbf{p})=\theta(\tau) \mathrm{e}^{-\left|p^{2} / 2 m-\varepsilon_{F}\right| \tau} .
$$




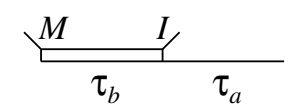

FIG. 7: "Normalization" diagram. It is the simplest diagram with the worm; its weight is a product of $G_{\downarrow}^{(0)}\left(\tau_{a}\right)$ and $\Gamma\left(\tau_{b}\right)$.

To simplify the description of updates below we will generically refer to $G_{\downarrow}^{(0)}$ - and $\Gamma$-propagators as backbone lines (BBLs) and denote them as $D$. The diagram order $N$ is given by the total number of spin-down propagators. We also use special counters to characterize the topology of the diagram. For each BBL we define a cover number, $N_{c}$, equal to the total number of $\tilde{G}$-lines covering a given BBL. A backbone line with $N_{c}=0$ is called uncovered. Finally, physical diagrams - the ones without worms - are divided into polaron (0) and molecule (1) sectors; the diagram sector is defined by the difference between the number of particle and hole spin-up propagators covering any of the $G_{\downarrow}^{(0)}$-lines (the same result is obtained by analyzing propagators covering $\Gamma$-lines after adding unity for the spin-up particle participating in the ladder diagrams).

\section{Updates}

The cyclic structure of diagrams in combination with the possibility of considering non-physical diagrams allows one to construct a very simple ergodic set of updates. The minimal set consists of two complementary pairs, Insert/Delete and Open/Close, and one self-complementary update Reconnect. The description that follows aims at the most transparent and straightforward realization of updates. Standard performance enhancement tricks and optimization protocols are not mentioned. In particular, we base our considerations on the updating scheme which may propose a change leading to a forbidden configuration. Such proposals are rejected as if they result in zero acceptance ratio.

Insert: This update applies only to physical-no worms - diagrams and is rejected otherwise. First, consider the polaron sector. Select at random one of the $G^{(0)}$ propagators; if it is covered, the update is automatically rejected. If the selected propagator is uncovered, insert a pair of new propagators, $\Gamma\left(\tau_{1}, \mathbf{p}\right)$ and $G_{\downarrow}^{(0)}\left(\tau_{2}, \mathbf{p}\right)$, right after the selected one. The new $\Gamma\left(\tau_{1}, \mathbf{p}\right)$-propagator is supposed to contain $\mathcal{I}$ and $\mathcal{M}$ at its ends. Worms radically simplify this diagram-order increasing update since due to conservation laws the momenta of new BBLs are equal to the global momentum of the diagram p. The times $\tau_{1}$ and $\tau_{2}$ are drawn from normalized probability distributions $W_{\Gamma}\left(\tau_{1}\right)$ and $W_{\downarrow}\left(\tau_{2}\right)$ (arbitrary at this point). Note that $W_{\Gamma}\left(\tau_{1}\right)$ and $W_{\downarrow}\left(\tau_{2}\right)$ can depend on $\mathbf{p}$ as a parameter. The acceptance ratio for this update is

$$
P_{\mathrm{ins}}=N C_{N+1} \frac{\Gamma\left(\tau_{1}, \mathbf{p}\right) G_{\downarrow}^{(0)}\left(\tau_{2}, \mathbf{p}\right)}{W_{\Gamma}\left(\tau_{1}\right) W_{\downarrow}\left(\tau_{2}\right)},
$$

where $C_{N}$ is an artificial weighing factor ascribed to all worm diagrams of order $N$ (it can be used for optimization purposes and depend on $\mathbf{p}$ too). A natural choice for $W$-functions is to make them proportional to BBL, i.e.

$$
W_{\Gamma}(\tau)=\frac{\Gamma(\tau, \mathbf{p})}{\int \Gamma\left(\tau^{\prime}, \mathbf{p}\right) d \tau^{\prime}}, W_{\downarrow}(\tau)=\frac{G_{\downarrow}^{(0)}(\tau, \mathbf{p})}{\int G_{\downarrow}^{(0)}\left(\tau^{\prime}, \mathbf{p}\right) d \tau^{\prime}} .
$$

Then, to have an acceptance ratio of order unity and independent of $\mathbf{p}$ we choose

$$
C_{N}=\frac{1}{N \Lambda}, \quad \Lambda=\int \Gamma\left(\tau_{1}, \mathbf{p}\right) d \tau_{1} \int G_{\downarrow}^{(0)}\left(\tau_{2}, \mathbf{p}\right) d \tau_{2} .
$$

In the rest of the paper, we will refer to this choice of $W$ and $C_{N}$ as "optimized", though we do not mean that it is the best one possible for the entire scheme. For the "optimized" choice

$$
P_{\text {ins }}=N /(N+1) .
$$

In the molecule sector, we essentially repeat all steps, up to minor modifications. Now the propagator being selected is $\Gamma$ (once again, the update is rejected if the selected propagator is covered.) Then, a pair of new propagators, $G_{\downarrow}^{(0)}\left(\tau_{1}, \mathbf{p}\right)$ and $\Gamma\left(\tau_{2}, \mathbf{p}\right)$, is inserted in front of the selected uncovered propagator. The new propagator $\Gamma\left(\tau_{2}, \mathbf{p}\right)$ inherits the outgoing spin-up line previously connected to the selected $\Gamma$; the latter gets instead the $\mathcal{M}$ end of the worm while the $\mathcal{I}$-end is attached to the new $\Gamma$. The acceptance ratio is identical to (28) [for the optimized choice it is $N /(N+1)$ ]. The polaron and molecule sectors are mutually exclusive due to particle conservation, thus only one type of the Insert update is applicable to a given diagram.

Delete: This update converts worm diagrams to physical ones while reducing the diagram order. It applies only to diagrams of order $N>1$ with worms being separated by one uncovered BBL. Otherwise, the update is rejected. If worms are separated by an uncovered BBL, then the left and right neighbor BBLs are also uncovered, and an update opposite to Insert is possible. In Delete we simply remove two consecutive BBL and worms from the diagram. Its acceptance probability is the inverse of Eq. (28),

$$
P_{\text {del }}=\frac{1}{(N-1) C_{N}} \frac{W_{\Gamma}\left(\tau_{1}\right) W_{\downarrow}\left(\tau_{2}\right)}{\Gamma\left(\tau_{1}, \mathbf{p}\right) G_{\downarrow}^{(0)}\left(\tau_{2}, \mathbf{p}\right)} .
$$

With Eqs. (29), (30), we have

$$
P_{\text {del }}=N /(N-1) . \quad \text { (optimized) }
$$


Close: The update applies only to diagrams with the worms. The proposal is to connect $\mathcal{I}$ and $\mathcal{M}$ with a line $\tilde{G}(\tau, \mathbf{q})$ and eliminate worms from the diagram. The momentum variable $\mathbf{q}$ is drawn from the probability distribution $W_{\uparrow}(\mathbf{q})$, while $\tau$ is the time interval between $\mathcal{I}$ and $\mathcal{M}$ to be covered by the new propagator. There are two ways of connecting $\mathcal{I}$ and $\mathcal{M}$, forwards and backwards. The ambiguity is automatically resolved by the absolute value of the momentum variable $\mathbf{q}$ : if $q \geq p_{F}\left(q<p_{F}\right)$, the propagator is supposed to go forwards (backwards). In practice, we first select the direction (with equal probabilities), and then generate the momentum variable $q$ accordingly: either $q \geq p_{F}$ or $q<p_{F}$.

The acceptance ratio for this update is

$$
P_{\mathrm{cl}}=\frac{2}{(2 \pi)^{3} N C_{N}} \frac{\tilde{G}(\tau, \mathbf{q})}{W_{\uparrow}(\mathbf{q})} \prod_{\nu} \frac{D_{\nu}\left(\tau_{\nu}, \mathbf{p}_{\nu}^{\prime}\right)}{D_{\nu}\left(\tau_{\nu}, \mathbf{p}_{\nu}\right)},
$$

where the subscript $\nu$ runs through all BBLs to be covered by the new propagator (clearly, $\tau=\sum_{\nu} \tau_{\nu}$ ). Primes indicate new values of the corresponding momenta:

$$
\mathbf{p}_{\nu}^{\prime}=\mathbf{p}_{\nu}-\mathbf{q} .
$$

As usual, the distribution function $W_{\uparrow}(\mathbf{q})$ can depend on $\tau$ and the direction of the propagator. The natural choice for this function would be

$$
\begin{gathered}
W_{\uparrow}(\mathbf{q})=\frac{\tilde{G}(\tau, \mathbf{q})}{\Omega(\tau)}, \\
\Omega(\tau)= \begin{cases}\int_{q \geq p_{F}} \tilde{G}_{\uparrow}(\tau, \mathbf{q}) d \mathbf{q} & \text { (forward), } \\
\int_{q<p_{F}} \tilde{G}_{\uparrow}(\tau, \mathbf{q}) d \mathbf{q} & \text { (backward), }\end{cases}
\end{gathered}
$$

leading to the optimized acceptance ratio

$$
P_{\mathrm{cl}}=\frac{2 \Lambda \Omega(\tau)}{(2 \pi)^{3}} \prod_{\nu} \frac{D_{\nu}\left(\tau_{\nu}, \mathbf{p}_{\nu}^{\prime}\right)}{D_{\nu}\left(\tau_{\nu}, \mathbf{p}_{\nu}\right)}
$$

In this Section we deal with diagrams based on bare propagators. To avoid double-counting, we have to exclude all cases which can be reduced to ladders already summed in $\Gamma$ 's. When $\mathcal{M}$ and $\mathcal{I}$ are on the nearestneighbor BBL the proposal to connect them with the spin-up particle propagator is rejected. The last rule to be monitored it to restrict all physical diagrams to be either in the polaron or molecule sectors, i.e. sectors different from 0 and 1 are not allowed.

Open: The update applies only to physical diagrams and proposes to create a worm by selecting at random and removing one of the spin-up propagators. The acceptance ratio is given by the inverse of (34), (38)

$$
P_{\mathrm{op}}=\frac{(2 \pi)^{3} N C_{N}}{2} \frac{W_{\uparrow}(\mathbf{q})}{\tilde{G}_{\uparrow}(\tau, \mathbf{q})} \prod_{\nu} \frac{D_{\nu}\left(\tau_{\nu}, \mathbf{p}_{\nu}^{\prime}\right)}{D_{\nu}\left(\tau_{\nu}, \mathbf{p}_{\nu}\right)},
$$

where the subscript $\nu$ runs through all BBLs covered by the propagator, $\tau=\sum_{\nu} \tau_{\nu}$, and $\mathbf{q}$ is the momentum of the selected spin-up propagator. Primes indicate new values of the BBL momenta:

$$
\mathbf{p}_{\nu}^{\prime}=\mathbf{p}_{\nu}+\mathbf{q} .
$$

In the optimized version, we have

$$
P_{\mathrm{op}}=\frac{(2 \pi)^{3}}{2 \Lambda \Omega(\tau)} \prod_{\nu} \frac{D_{\nu}\left(\tau_{\nu}, \mathbf{p}_{\nu}^{\prime}\right)}{D_{\nu}\left(\tau_{\nu}, \mathbf{p}_{\nu}\right)} .
$$

Reconnect: The purpose of this update is to change the topology of diagrams with the worm. The proposal is to select at random one of the $\tilde{G}$-propagators and swap its outgoing end with $\mathcal{M}$; the momentum of the propagator remains the same, only its time variable changes from $\tau_{0}$ to $\tau_{0}^{\prime}$. The acceptance ratio is given by:

$$
P_{\text {rec }}=\frac{\tilde{G}\left(\tau_{0}^{\prime}, \mathbf{q}\right)}{\tilde{G}\left(\tau_{0}, \mathbf{q}\right)} \prod_{\nu} \frac{D_{\nu}\left(\tau_{\nu}, \mathbf{p}_{\nu}^{\prime}\right)}{D_{\nu}\left(\tau_{\nu}, \mathbf{p}_{\nu}\right)} .
$$

The subscript $\nu$ runs through all BBLs that will change their momenta (and cover numbers $N_{c}$ 's) as a result of the update. Topologically, there are two different situations (the two a complementary to each other in terms of the update): (i) $\mathcal{M}$ is covered by the propagator in question, and (ii) $\mathcal{M}$ is not covered by the propagator. Correspondingly, the new values of the diagram variables are calculated as

$$
\left(\tau_{0}^{\prime}, \mathbf{p}_{\nu}^{\prime}\right)= \begin{cases}\left(\tau_{0}-\tau, \mathbf{p}_{\nu}+\mathbf{q}\right) & (\mathrm{i}) \\ \left(\tau_{0}+\tau, \mathbf{p}_{\nu}-\mathbf{q}\right) & (\mathrm{ii})\end{cases}
$$

with $\tau=\sum_{\nu} \tau_{\nu}$.

The above set of updates is ergodic. It can be supplemented by additional updates that may improve the algorithm performance by more efficient sampling of the diagram variables and topologies. Over-complete sets of updates are also useful for meaningful tests of the detailed balance. The possibilities are endless, and here we simply mention two updates we have been using.

Time shift: We propose new time variables, $\tau_{\nu} \rightarrow \tau_{\nu}^{\prime}$, for all uncovered BBLs (labeled here with the subscript $\nu)$. The acceptance probability is

$$
P_{\mathrm{sh}}=\prod_{\nu} \frac{W_{\nu}\left(\tau_{\nu}, \mathbf{p}\right) D_{\nu}\left(\tau_{\nu}^{\prime}, \mathbf{p}\right)}{W_{\nu}\left(\tau_{\nu}^{\prime}, \mathbf{p}\right) D_{\nu}\left(\tau_{\nu}, \mathbf{p}\right)} .
$$

All uncovered propagators have the same momentum $\mathbf{p}$. With the optimized choice for $W_{\nu}\left(\tau_{\nu}, \mathbf{p}\right) \propto D_{\nu}\left(\tau_{\nu}, \mathbf{p}\right)$ the acceptance ratio becomes unity.

Redirect: Here we propose to select at random one of the $\tilde{G}$-propagators and change its direction to the opposite. Simultaneously, we change the momentum of the selected propagator (resulting in new momenta for all BBL it covers or will cover as a result of the update). Let the selected propagator be $\tilde{G}(\tau, \mathbf{q})$, and the new one be $\tilde{G}\left(\tau^{\prime}, \mathbf{q}^{\prime}\right)$ with $\tau=\sum_{\nu} \tau_{\nu}$, and $\tau^{\prime}=\sum_{\lambda} \tau_{\lambda}$, where $\nu$ runs through all BBLs covered by the propagator $\tilde{G}_{\uparrow}(\tau, \mathbf{q})$ and $\lambda$ runs through all BBLs to be covered by the propagator 


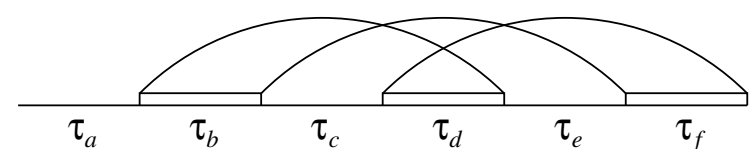

FIG. 8: A $G \Sigma$-diagram contributing to the polaron selfenergy. It factorizes into a product of $G_{\downarrow}^{(0)}\left(\tau_{a}\right)$ and $\Sigma(\tau=$ $\left.\tau_{b}+\tau_{c}+\tau_{d}+\tau_{e}+\tau_{f}\right)$

$\tilde{G}_{\uparrow}\left(\tau^{\prime}, \mathbf{q}^{\prime}\right)$. We assume that $\mathbf{q}^{\prime}$ is drawn from the distribution $W_{\uparrow}$ introduced above. In this case, the acceptance ratio is given by

$$
\begin{aligned}
& P_{\mathrm{rdr}}= \frac{W_{\uparrow}(\tau, \mathbf{q}) \tilde{G}\left(\tau^{\prime}, \mathbf{q}^{\prime}\right)}{W_{\uparrow}\left(\tau^{\prime}, \mathbf{q}^{\prime}\right) \tilde{G}(\tau, \mathbf{q})} \times \\
& {\left[\prod_{\nu} \frac{D_{\nu}\left(\tau_{\nu}, \mathbf{p}_{\nu}^{\prime}\right)}{D_{\nu}\left(\tau_{\nu}, \mathbf{p}_{\nu}\right)}\right]\left[\prod_{\lambda} \frac{D_{\lambda}\left(\tau_{\lambda}, \mathbf{p}_{\lambda}^{\prime}\right)}{D_{\lambda}\left(\tau_{\lambda}, \mathbf{p}_{\lambda}\right)}\right], } \\
& \mathbf{p}_{\nu}^{\prime}=\mathbf{p}_{\nu}+\mathbf{q}, \quad \mathbf{p}_{\lambda}^{\prime}=\mathbf{p}_{\lambda}-\mathbf{q}^{\prime} .
\end{aligned}
$$

For the optimized choice of $W_{\uparrow}$, see Eq. (36),

$$
\frac{W_{\uparrow}(\tau, \mathbf{q}) \tilde{G}\left(\tau^{\prime}, \mathbf{q}^{\prime}\right)}{W_{\uparrow}\left(\tau^{\prime}, \mathbf{q}^{\prime}\right) \tilde{G}(\tau, \mathbf{q})} \rightarrow \frac{\Omega\left(\tau^{\prime}\right)}{\Omega(\tau)} .
$$

Diagram sign: The sign of a diagram with worms is somewhat arbitrary since it is not physical. The ambiguity is resolved by assuming that $\mathcal{M}$ is always connected to $\mathcal{I}$ in the backward direction by an auxiliary unity propagator. Then, to comply with the diagrammatic rules, we change the configuration sign each time any of the following updates are accepted: (i) Reconnect, (ii) Open/Close updates dealing with spin-up propagators in the forward direction, (iii) Insert/Delete in the molecule sector, and (iv) Redirect. For Open/Close updates dealing with spin-up propagators in the backward direction the sign remains the same, because here the sign coming from changing the number of closed spin-up loops is compensated by the sign in Eq. (26); the same is also true for Insert/Delete updates in the polaron sector (due to our choice of the auxiliary propagator sign). For precisely the same sign compensation reason, the Redirect update does change the sign despite the fact that it preserves the number of loops.

\section{E. Estimators}

Only physical diagrams with one uncovered $G_{\downarrow}^{(0)}$ propagator contribute to the polaron self-energy. We will call them $G \Sigma$-diagrams. An example is shown in Fig. 8 , [Depending on restrictions imposed on the configuration space (easy to implement in any scheme) physical diagrams with more than one uncovered $G_{\downarrow}^{(0)}$ propagator are

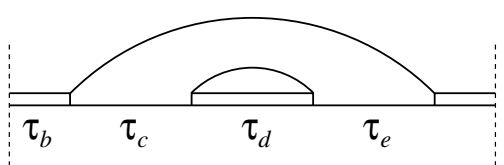

FIG. 9: A $\Gamma \tilde{K}$-diagram contributing to the molecule selfenergy. It factorizes into a product of $\Gamma\left(\tau_{b}\right)$ and $\tilde{K}(\tau=$ $\tau_{c}+\tau_{d}+\tau_{e}$ ). The vertical dashed lines cut (for the sake of better visual perception) the same $\Gamma\left(\tau_{b}\right)$-line.

either filtered out at the time when the contribution to the self-energy histogram is made, or, are not produced in the simulation at all.] They factorize into a product of $G_{\downarrow}^{(0)}$ and some diagram contributing to the self-energy $\Sigma$. The utility of cyclic representation is that the uncovered propagator can be anywhere on the backbone. In view of factorization, it is easy to write the MC estimator for the integral (to simplify notations we omit irrelevant to the discussion momentum $\mathbf{p}$ )

$$
I=\int_{0}^{\infty} f(\tau) \Sigma(\tau) d \tau
$$

where $f(\tau)$ is some function [see, e.g., Eqs. (13), (14)]. Indeed, consider the estimator

$$
\mathcal{E}_{I}=\delta_{G \Sigma} f(\tau)
$$

which counts all instances of $G \Sigma$-diagrams with an additional weight $f(\tau)$. Here $\delta_{G \Sigma}$ is unity for each $G \Sigma$ diagram and zero otherwise, and $\tau$ is the total duration in time of the $\Sigma$-part of the $G \Sigma$-diagram. The Monte Carlo average of this estimator is

$$
\left\langle\mathcal{E}_{I}\right\rangle_{\mathrm{MC}} \propto \int_{0}^{\infty} G_{\downarrow}^{(0)}\left(\tau^{\prime}\right) d \tau^{\prime} \int_{0}^{\infty} f(\tau) \Sigma(\tau) d \tau .
$$

Similarly, within the same scheme, we collect statistics of all "normalization" diagrams, see Fig. [7 using an estimator projecting to the first-order diagram with the worm, $\delta_{\text {norm }}$. Then,

$$
\left\langle\delta_{\text {norm }}\right\rangle_{\mathrm{MC}} \propto C_{1} \int_{0}^{\infty} G_{\downarrow}^{(0)}\left(\tau^{\prime}\right) d \tau^{\prime} \int_{0}^{\infty} \Gamma(\tau) d \tau .
$$

The proportionality coefficient cancels in the ratio of the two averages, leading to

$$
I=C_{1} \frac{\left\langle\mathcal{E}_{I}\right\rangle_{\mathrm{MC}}}{\left\langle\delta_{\mathrm{norm}}\right\rangle_{\mathrm{MC}}} \int_{0}^{\infty} \Gamma(\tau) d \tau .
$$

In particular, for the optimal choice of $C_{N}$, we have

$$
I=\frac{\left\langle\mathcal{E}_{I}\right\rangle_{\mathrm{MC}}}{\left\langle\delta_{\text {norm }}\right\rangle_{\mathrm{MC}}}\left[\int_{0}^{\infty} G_{\downarrow}^{(0)}(\tau) d \tau\right]^{-1} .
$$

Imaginary-time integrals for the product of $\Sigma(\tau)$ and exponentials, see Eqs. (13) and (14), is all we need to 
determine the polaron energy and residue. For the boldline generalization of the scheme described in the next Section we need the entire dependence of self-energy on time and momentum. This is achieved by differentiating partial contributions of $G \Sigma$-diagrams. For example,

$$
\mathcal{E}_{\Sigma, i}=\delta_{G \Sigma} \delta_{\tau \in \text { bin }_{i}}
$$

is an estimator counting contributions with $\tau$ within the $i$-th imaginary-time bin of width $\Delta \tau_{i}$ centered at the point $\tau_{i}$. Due to linear relation between $I$ and $\Sigma(\tau)$ we immediately realize that (for optimized choice)

$$
\Sigma\left(\tau_{i}\right)=\frac{\left\langle\mathcal{E}_{\Sigma, i}\right\rangle_{\mathrm{MC}}}{\left\langle\delta_{\mathrm{norm}}\right\rangle_{\mathrm{MC}}}\left[\Delta \tau_{i} \int_{0}^{\infty} G_{\downarrow}^{(0)}(\tau) d \tau\right]^{-1}
$$

In complete analogy with the polaron case, we consider $\Gamma \tilde{K}$-diagrams that contain one, and only one, uncovered $\Gamma$-propagator, see Fig. 9, and use them to collect statistics for the molecule self-energy. Up to straightforward replacements $G_{\downarrow}^{(0)} \leftrightarrow \Gamma, \Sigma \leftrightarrow \tilde{K}$ all relations of this subsection hold true.

\section{SIMULATING $T$-MATRIX $\Gamma(\tau, p)$ BY BOLD DIAGRAMMATIC MONTE CARLO}

Despite relatively simple form of Eqs. (23) and (24), tabulating the two-dimensional function $\Gamma(\tau, p)$ with high accuracy using the inverse Laplace transform of $\Gamma(\omega, p)$ turns out to be a time consuming job. In this work we have used an alternative route based on the bold diagrammatic Monte Carlo technology introduced recently in Ref. [32]. The crucial observation is that the $T$-matrix $\Gamma(\tau, p)$ can be diagrammatically related to its vacuum counterpart $\Gamma_{0}(\tau, p)$, see Fig. 10, with the latter is known analytically:

$$
\Gamma_{0}(\tau, p)=\frac{4 \pi}{m^{3 / 2}} e^{\left(\epsilon_{F}+\mu-p^{2} / 4 m\right) \tau} g_{\mp}(\tau)
$$

were

$$
g_{\mp}(\tau)=-\frac{1}{\sqrt{\pi \tau}} \pm \sqrt{E} e^{E \tau} \operatorname{erfc}( \pm \sqrt{E \tau})
$$

for negative/positive values of the scattering length, $E=$ $1 / m a^{2}$, and $\operatorname{erfc}(x)$ is the error-function. [The Fermienergy and the chemical potential in Eq. (56) come from the global energy shift necessary for compliance with the Dyson equation shown in Fig. 10.]

With the explicit expression for the product of two vacuum propagators the relation shown in Fig. 10 reads (the momentum argument of $\Gamma(\tau, p)$ is suppressed for clarity)

$$
\begin{aligned}
& -\Gamma(\tau)=-\Gamma_{0}(\tau)+\int_{0}^{\tau} d s \int_{s}^{\tau} d s^{\prime} \Gamma_{0}(s) \Gamma\left(\tau-s^{\prime}\right) \\
& \times \int_{q<k_{F}} \frac{d \boldsymbol{q}}{(2 \pi)^{3}}\left(-e^{-\left[(\boldsymbol{p}-q)^{2}+\boldsymbol{q}^{2}\right]\left(s^{\prime}-s\right) / 2 m}\right) .
\end{aligned}
$$

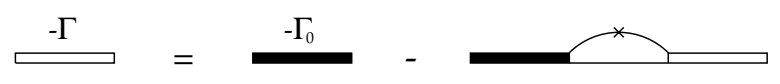

FIG. 10: Diagrammatic equation for the $T$-matrix $\Gamma(\tau, p)$. The arc is the vacuum spin-up propagator with the constraint $q<k_{F}$ on its momentum $q$. The meaning of this equation is nothing but correcting the vacuum result $\Gamma_{0}$ by subtracting contributions from the spin-up fermions with momenta $q<$ $k_{F}$.

Equation (58) is one of the simplest examples of problems solvable by bold diagrammatic Monte Carlo 32. We refer the reader to Ref. 32], where the algorithm of solving such equations is described in detail. Here we just mention some specific details. We find it helpful to start from a good trial function for obtaining high-accuracy results in a short simulation time. We achieve this goal using the following protocol. We start the simulation by restricting imaginary time to be smaller than $\tau_{\max }$ and select relatively short $\tau_{\max }$. When the result is accurate enough, we extrapolate it to longer times, increase $\tau_{\max }$, and restart the simulation with the extrapolated function, $\Gamma_{\text {ext }}$ serving as the trial function, i.e. we substitute $\Gamma=\Gamma_{\text {ext }}+\delta \Gamma$ to Eq. (58) and solve for $\delta \Gamma$. If necessary, this procedure can be repeated several times.

\section{SIMULATION RESULTS AND SERIES CONVERGENCE PROPERTIES}

Nearly all results in this paper were obtained by simulating diagrams built on bare one- and two-particle propagators $G_{\downarrow}^{(0)}$ and $\Gamma$. We observed that the corresponding series are likely to be divergent. This, however, does not mean that the entire idea of calculating contributions from diagrams of higher and higher order and extrapolating results to the infinite order is useless and ill-defined. On the contrary, it was recognized long ago that appropriate re-summation techniques allow one to determine reliably the function standing behind the divergent series. Moreover, all re-summation techniques (formally, there are infinitely many!), if applicable, have to agree with each other on the final result. This important observation vastly increases the utility of the Diag-MC technique we are developing here. In the next Section we demonstrate that making the series for $\Sigma$ self-consistent with the use of Dyson equation - bold-line technique - is another way to improve series convergence properties.

For the resonant Fermi-polaron considered here the Cesàro-Riesz summation method solves the convergence problem. In general, for any quantity of interest - in our case they are polaron or molecule self-energy - one constructs partial sums

$$
\Sigma\left(N_{*}\right)=\sum_{N=1}^{N_{*}} D_{N} F_{N}^{\left(N_{*}\right)}
$$




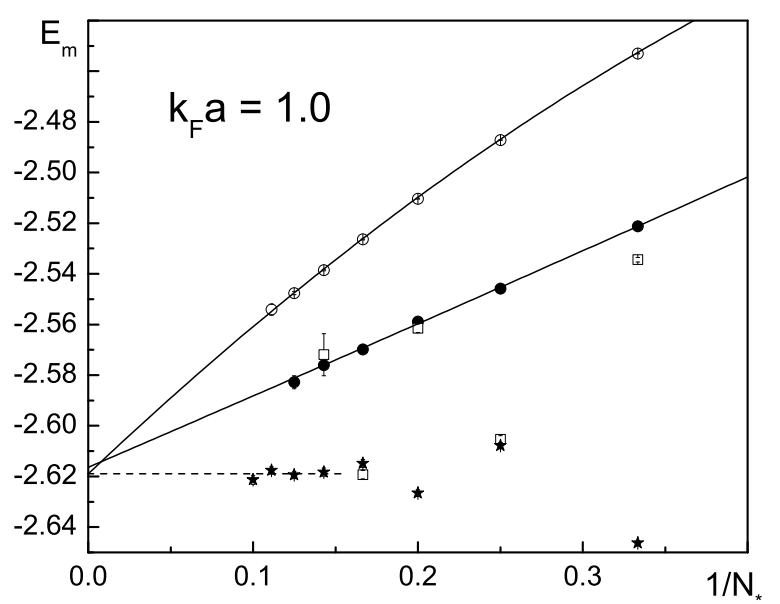

FIG. 11: The molecule energy (at $k_{F} a=1$ ) as a function of the maximum diagram order $N_{*}$ for different summation techniques: Cesàro (open squares), Riesz $\delta=2$ (filled circles, fitted with the parabola $\left.y=-2.6164+0.28013 x+0.01638 x^{2}\right)$, Riesz $\delta=4$ (open circles, fitted with the parabola $y=$ $-2.6190+0.61635 x-0.3515 x^{2}$ ), and Eq. (61) (stars fitted with the horizontal dashed line). Reproduced from Ref. [12].

defined as sums of all terms up to order $N_{*}$ with the $N$ th order terms being multiplied by the factor $F_{N}^{\left(N_{*}\right)}$. In the limit of large $N_{*}$ and $N \ll N_{*}$ the multiplication factors $F$ approach unity while for $N \rightarrow N_{*}$ they suppress higher-order contributions in such a way that $\Sigma\left(N_{*}\right)$ has a well-defined $N_{*} \rightarrow \infty$ limit. There are infinitely many ways to construct multiplication factors satisfying these conditions. This immediately leads to an important consistency check: Final results have to be independent of the choice of $F$. In the Cesàro-Riesz summation method we have

$$
F_{N}^{\left(N_{*}\right)}=\left[\left(N_{*}-N+1\right) / N_{*}\right]^{\delta}, \quad \text { (Cesàro-Riesz) . }
$$

Here $\delta>0$ is an arbitrary parameter $(\delta=1$ corresponds to the Cesàro method). The freedom of choosing the value of Riesz's exponent $\delta$ can be used to optimize the convergence properties of $\Sigma\left(N_{*}\right)$.

We proceed as follows. For the series truncated at order $N_{*}$ we first determine the polaron and molecule energies and then study their dependence on $N_{*}$ as $N_{*} \rightarrow \infty$. In Fig. 11 we show results for the molecule energy at $k_{F} a=1$. Without re-summation factors the data are oscillating so strongly that any extrapolation to the infinite diagram order would be impossible; we consider this as an indication that the original series are divergent. Oscillations remain pronounced for $\delta=1$, but are strongly suppressed for larger values of $\delta$, so that for $\delta=4$ we were not able to resolve odd-even oscillations any more. However, the smoothness of the curve for large $\delta=4$ comes at the expense of increased curvature, which renders the extrapolation to the $1 / N_{*} \rightarrow 0$ limit more vulnerable to systematic errors. Empirically, we constructed a factor $F_{N}^{\left(N_{*}\right)}$ which leads to a faster convergence [see an example

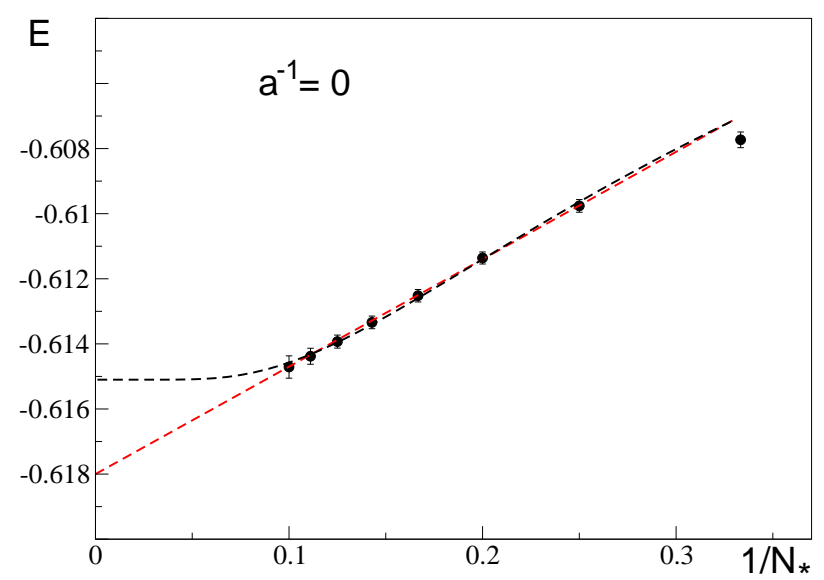

FIG. 12: (Color online) The polaron energy (at the unitarity point $a^{-1}=0$ ) as a function of the maximum diagram order $N_{*}$ using Eq. 61). The data are fitted using linear $-0.618+0.033 / N_{*}(\mathrm{red})$ and exponential $-0.6151+$ $0.026 e^{-0.39 N_{*}}$ (black) functions to have an estimate of systematic errors introduced by the extrapolation procedure.

in Fig. [11)]:

$$
F_{N}^{\left(N_{*}\right)}=C^{\left(N_{*}\right)} \sum_{m=N}^{N_{*}} \exp \left[-\frac{\left(N_{*}+1\right)^{2}}{m\left(N_{*}-m+1\right)}\right],
$$

where $C^{\left(N_{*}\right)}$ is such that $F_{1}^{\left(N_{*}\right)}=1$. The most important conclusion we draw from Fig. 11] is that in our case the series are subject to re-summation methods and the result of extrapolation is method independent. We consider small variations in the final answer due to different re-summation techniques and extrapolation methods as our systematic errors. An example is shown in Fig. 12 , In the next section we will present evidence that the actual answer is closer to the upper bound of -0.615 . We see that in the absence of additional information one has to allow for different ways of extrapolating the answer.

Apart from consistency checks, one can test numerical results against an analytic prediction for the strong coupling limit $k_{F} a \rightarrow 0$ corresponding to a compact molecule scattering off majority spins. In this limit the molecule energy is given by the expression

$$
E_{m}=-\frac{1}{m a^{2}}-\varepsilon_{F}+\frac{2 \pi \tilde{a}}{(2 / 3) m} n_{\uparrow} \quad\left(k_{F} a \rightarrow 0\right),
$$

where the first term is the molecule binding energy in vacuum, the second term reflects finite chemical potential of spin-up fermions, and the last term comes from the interaction between the composite molecule with the Fermi gas. The molecule-fermion $s$-scattering length $\tilde{a} \approx 1.18 a$ $[35,36]$ is obtained from the non-perturbative solution of the three-body problem. Agreement with Eq. (62) provides a robust test for the entire numerical procedure of sampling asymptotic diagrammatic series. Our data are in a perfect agreement with the $\tilde{a} \approx 1.18 a$ result within 

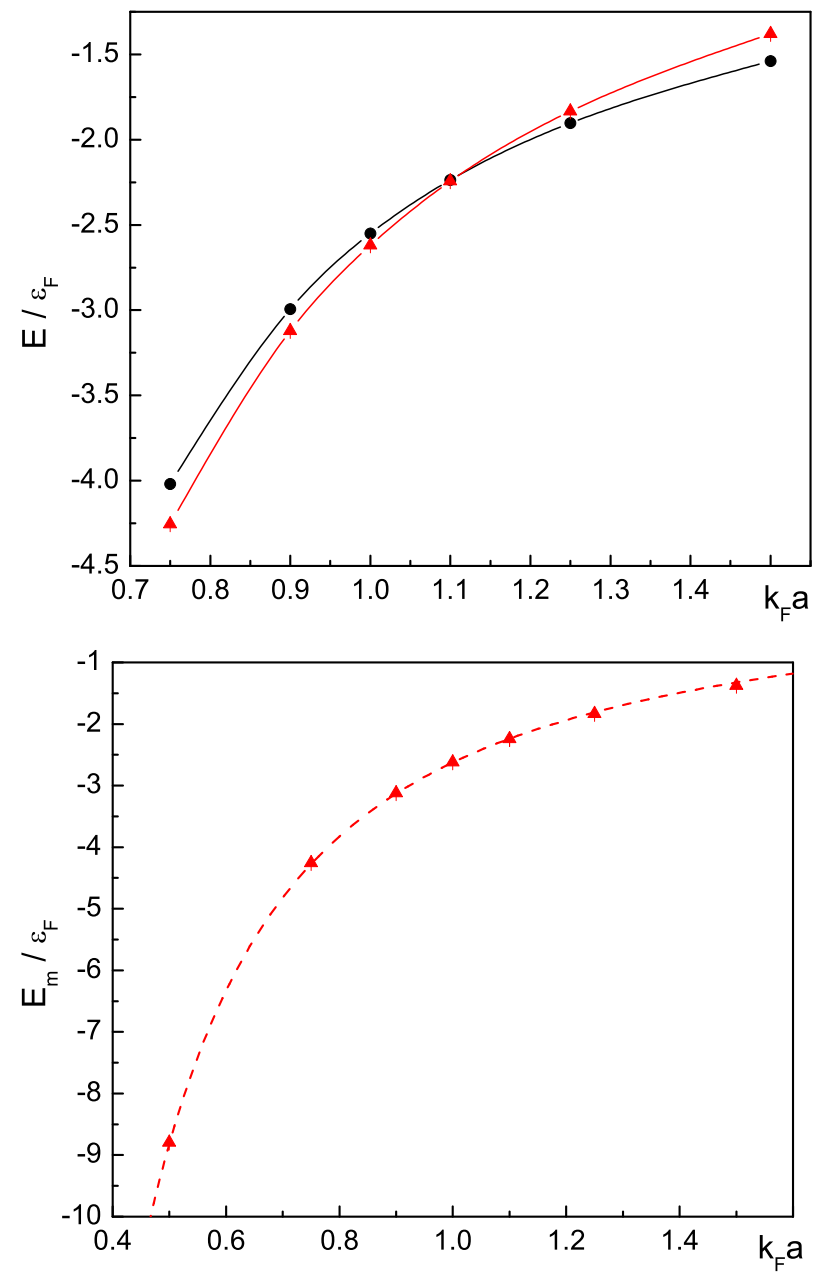

FIG. 13: (Color online) Polaron (black circles) and molecule (red triangles) energies (in units of $\varepsilon_{F}$ ) as functions of $k_{F} a$. The dashed line on the lower panel corresponds to Eq. (62). Reproduced from Ref. [12].

the statistical uncertainty of the order of $5 \%$, see the lower panel in Fig. 13 .

Somewhat surprising outcome is that $E_{m}$ is described by Eq. (62) very accurately all the way to the crossing point. This fact can be used to approximate the energy density functional of the superfluid polarized phase in the strongly imbalanced gas for $k_{F} a<1$ as that of the miscible dilute molecule gas coupled to spin-up fermions [19] (see also Refs. [39, 40])

$$
E=\frac{3}{5} \epsilon_{F} n_{\uparrow}-\left[\frac{1}{m a^{2}}-\frac{2 \pi \tilde{a}}{(2 / 3) m} n_{\uparrow}\right] n_{\downarrow}+\frac{\pi a_{M M}}{m} n_{\downarrow}^{2},
$$

where $n_{\uparrow}, n_{\downarrow}$ are densities of unpaired spin-up fermions and molecules, and $a_{M M} \approx 0.6 a$ is the molecule-molecule scattering length [38]. Within this approach it is found that the system undergoes phase separation for $k_{F} a>$ 0.56 [19].

Phase separation precludes one from investigating the crossing point between the polaron and molecule curves

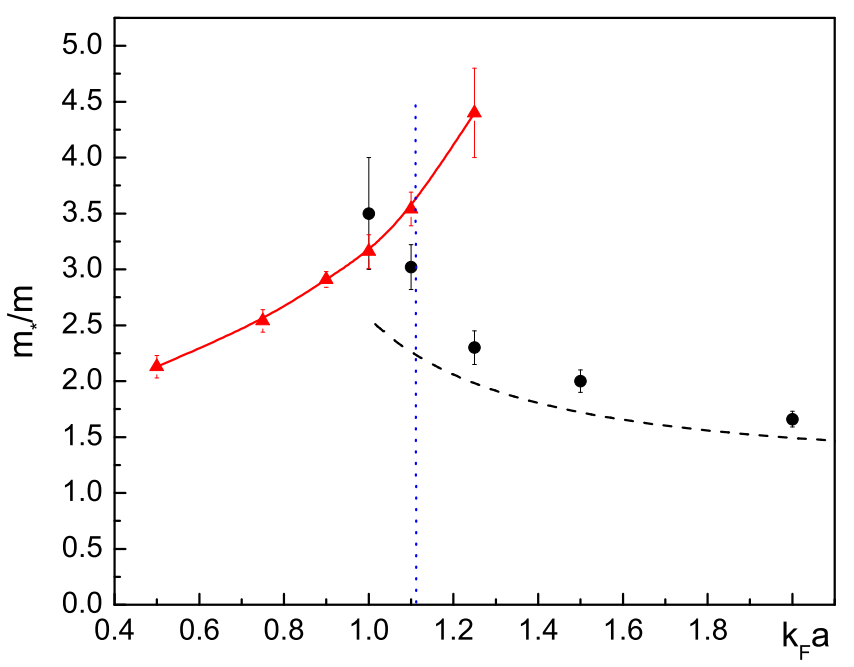

FIG. 14: (Color online) Polaron (black circles) and molecule (red triangles) effective mass as functions of $k_{F} a$. The vertical dotted line stands for $\left(k_{F} a\right)_{c}=1.11$. The dashed line is the contribution from the first diagram [37]. Reproduced from Ref. [12].

in trapped imbalanced Fermi gases. It also rules out the multi-critical point on the phase diagram predicted in Ref. [17]. This issues, however, are not directly relevant to our study of properties of one spin-down particle. In Fig. 13, we present polaron and molecule energies in the region of $k_{F} a \sim 1$ where the nature of the quasiparticle state changes. The crossing point is found to be at $\left(k_{F} a\right)_{c}=1.11(2)$. Overall, both curves are in excellent agreement with the variational Monte Carlo simulations [16, 19]. There is a certain degree of accidental coincidence in the fact that the polaron self-energy is nearly exhausted by the first-order diagram considered in Ref. [37], see also Fig. 12. As we show in the next section, both second-order and third-order diagrams make considerable contributions to the answer but they happen to nearly compensate each other, i.e. Green's function renormalization and vertex corrections have similar amplitudes and opposite signs.

The intersection of the polaron and molecule curves can be determined very accurately because both solutions are describing well-defined quasiparticles at the crossing point. This is because matrix elements connecting the two branches involve at least four particles and their on-resonance phase volume is zero at $\left(k_{F} a\right)_{c}$. Indeed, the energy, momentum, and particle number (for each spin direction) conservation laws dictate that polaron decays into molecule, two holes and one spin-up particle (molecule decays into polaron two spin-up particles and one hole). For this process the final-state phase volume gets negligibly small as compared to the energy difference $\left|E_{p}-E_{m}\right|$ at and in the vicinity of the crossing point.

The data for the effective mass is presented in Fig. 14. At the crossing point the effective mass curve is discontin- 
uous as expected for the exact crossing between two solutions. One important observation is that good agreement with Eq. (62) for molecule energy in the entire region $k_{F} a<1$ does not guarantee yet that the compact-boson approximation is accurate in the same region if properties other than energy are addressed. In this sense, even good agreement with Eq. (62) has to be taken with a grain of salt since (62) assumes that the molecule mass is $2 m$ and independent of $k_{F} a$. The actual effective mass is significantly enhanced in the vicinity of $\left(k_{F} a\right)_{c}$.

\section{NUMERIC REALIZATION OF THE "BOLD-LINE" TRICK}

The success of the Diag-MC method for fermi-polarons is, to large extent, due to small error bars we have for the sign-alternating sums of high-order diagrams. In general, it is expected that the computational complexity of getting small error bars for sign-alternating sums in the limit of large $N$ is exponential (or factorial) in $N$ since it usually scales with the configuration space volume. Any tricks that reduce the configuration space while keeping the scheme exact are worth investigating. In fact, we have already used one of such tricks above when we introduced $\Gamma$ summing up all ladder diagrams. As a result, the diagram order was defined by the number of $G_{\downarrow}$-lines, not bare interaction potential vertexes, and ladder-reducible diagrams were excluded from the configuration space.

In this Section we go one step further and apply another method, well-known in analytic calculations (but virtually never carried out analytically to high-order; for first-order diagrams it is known as the self-consistent Born approximation). It is called the "bold-line" trick. The relation between $G_{\downarrow}, G_{\downarrow}^{(0)}$ and $\Sigma$ accounts for the infinite sum of diagrams forming geometrical series. Now, if one-particle lines in self-energy diagrams are representing exact Green's function (in this case they are drawn in bold) then many diagrams have to be excluded to avoid double counting. Namely, any structure which can be interpreted locally as part of the Dyson equation for $G_{\downarrow}$ has to be crossed out. Though formally the diagram order is still defined by the number of $G_{\uparrow}$, it is in fact representing a whole class of diagrams (up to infinite order) in the original, or bare, terms. Clearly, the MC scheme is now self-consistently defined and potentially even finite number of "bold" diagrams can capture non-perturbative effects.

Recently, we have demonstrated that the bold-line trick is compatible with Diag-MC and the corresponding scheme has been termed the bold diagrammatic Monte Carlo (BMC) 32]. There are two routes for implementing the bold-line technique. The first one is to arrange two (running in parallel) coupled Monte Carlo processes: one sampling the series for the self-energy in terms of exact propagators, and the other one sampling propagators from the Dyson equation. The latter process is essentially the same as the process we use for pre-calculating $\Gamma$, with an important new feature that the self-energy used in the sampling is permanently updated. The second route is specific for Dyson-type equations which allow trivial algebraic solution in momentum representation. In the present work, we use the second route.

The implementation of the bold-line trick requires that we introduce an update which changes the global momentum $\mathbf{p}$ of the diagram. This update applies only to the simplest normalization diagrams, see Fig. 7. The integrated weight of normalization diagrams is given by $\Lambda(\mathbf{p})$, see Eqs. (30) and (51). In the update, we select the new value for the global momentum from the probability distribution $\Lambda(\mathbf{p})$ and propose new time variables for the spin-down and pair propagators from the optimized probability distributions $W_{\Gamma}\left(\tau_{1}, \mathbf{p}\right)$ and $W_{\downarrow}\left(\tau_{2}, \mathbf{p}\right)$. Since new variables are seeded using the exact diagram weight the acceptance ratio is unity. In practice, the modulus of the global momentum variable is defined on the discrete set of points.

We start the simulation with $G_{\downarrow}=G_{\downarrow}^{(0)}$, and collect statistics to the momentum-time histogram of $\Sigma$ from bold-line diagrams. After a certain number of updates, we perform fast Fourier transform of $\Sigma(\tau, p)$ to obtain $\Sigma(\omega, p)$, calculate $G_{\downarrow}(\omega, p)$ using Dyson equation, which is then transformed back to $G_{\downarrow}(\tau, p)$. The simulation proceeds as before with the $G_{\downarrow}(\omega, p)$ function being recalculated at regular time intervals to reflect additional statistics accumulated in $\Sigma$. Obviously, the self-consistent feedback present in the bold-line scheme at the beginning of the simulation violates the detailed balance equation each time the function $G_{\downarrow}$ is updated. Only in the long simulation time limit when both $\Sigma$ and $G$ do not change any more is the detailed balance satisfied.

The other point which requires special care is the treatment of ladder-reducible diagrams. In the bold-line implementation we have to allow ladder diagrams back, but each spin-down line in the ladder-reducible structure now has to be understood as a difference $G_{\downarrow}-G_{\downarrow}^{(0)}$. Indeed, ladder diagrams included in $\Gamma$ are built on bare propagators and thus have to be corrected for the difference between the bare and exact propagators.

Finally, we apply the bold-line approach in the molecule channel as well. In fact, the scheme was designed to be identical in the one- and two-particle sectors. Now, in all self-energy diagrams we have to substitute $G_{\downarrow}^{(0)}$ for $G_{\downarrow}$ and $\Gamma$ for $Q$, with both $G$ and $Q$ being periodically recalculated to reflect additional statistics statistics accumulated to the $\Sigma$ - and $\tilde{K}$-histograms. Correspondingly, diagrams which can be locally interpreted as part of the Dyson equation in the molecule sector have to be excluded. [Ironically, this means that ladder diagrams are not allowed once again with the exception for the first-order diagram in $\tilde{K}$ which ensures that ladders are built on $G_{\downarrow}$, see previous paragraph.] The updates and acceptance ratios do not change in the bold-line representation, but in the optimized version the probability distributions $W_{\downarrow}$ and $W_{\Gamma}$ (and their normalization integrals) are now proportional to $G_{\downarrow}$ and $Q$, i.e. they have 
to be changed each time the new solutions of the Dyson equations are generated.

As discussed in Ref. [32], the Monte Carlo procedure of solving self-consistent equations is more robust and has better convergence properties then standard iterations, especially for sign-alternating series. There are additional tools to improve the efficiency and convergence, some are self-explanatory. It definitely helps to start with the initial function $G_{\downarrow}$ as close as possible to the actual solution (the final answer should not depend on small variations of the initial choice). For example, the simulation for a given value of $k_{F} a$ may start with the final solution for the neighboring $k_{F} a$ point. The initial statistics has to be discarded or "erased" according to some protocol. If analytic expressions in special cases are available, e.g. in the perturbation theory or strong coupling limits, they can be used to match numeric data and restrict the parameter range probed in the simulation.

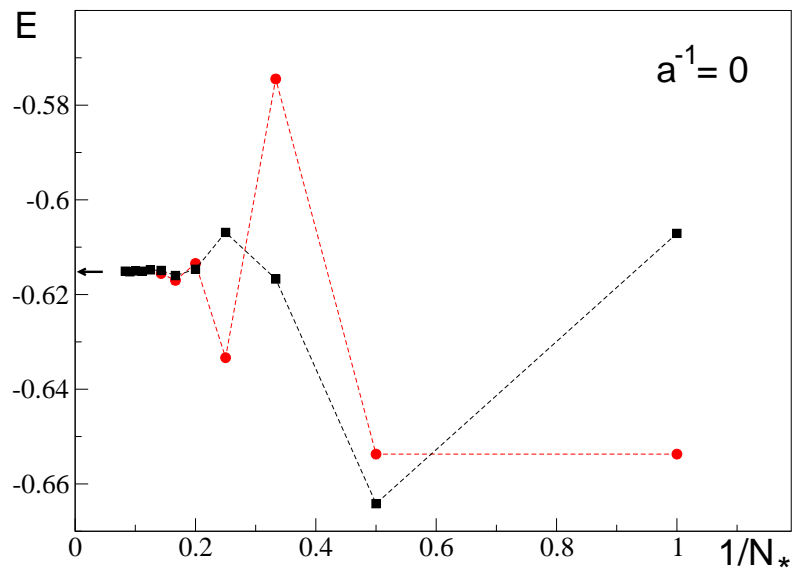

FIG. 15: (Color online) Polaron energy as a function of the maximum diagram order $N_{*}$ at the unitary point $k_{F} a=\infty$ within the bold-line approach. Black squares show results when the bold-line approach was implemented only for the polaron propagator. When both polaron and molecule propagators in diagrams are given by $G_{\downarrow}$ and $Q$ one obtains results shown by red circles.

In Fig. 15 we present data for the polaron energy at the unitary point calculated using the self-consistent scheme. As before, the diagram order is defined by the number of spin-down propagators. To see the difference between various approaches we first calculated $E$ with the bold-line trick implemented only for spin-down propagators (black squares) and then for both spin-down and molecule propagators (red circles). This plot makes it clear that the perturbation theory result [37] is accurate because corrections to spin-down propagators nearly cancel vertex corrections. Figure 15 is also telling the story which we observe happening over and over again for other strongly-correlated condensed matter problems: it does not really make any sense to propose "better" approxi- mations which account only for some incomplete set of diagrams. For example, if in the first-order diagram $\Gamma$ is replaced with $Q$ (self-consistent Born approximation in the molecule sector), the answer is getting much worse! Moreover, using exact expressions for $G_{\downarrow}$ and $Q$ in irreducible diagrams up to third-order (!) results in an oscillation (the highest circle in Fig. 15) which forces one to think that the final answer is probably even further up. Fortunately, with the bold-line Diag-MC technique developed in this article we can see how different approximations work and what their actual value is, term by term.

\section{CONCLUSIONS}

The sign-problem in MC simulations is the problem of obtaining small error-bars for system parameters which allow reliable extrapolation of results to the thermodynamic limit. Most MC schemes are based on simulations of finite-size systems (of linear size $L$ ) with the configuration space volume growing exponentially/factorially with $L^{3}$ and inverse temperature $1 / T$ (for quantum models). Since error bars grow with the configuration space volume they are completely out of control before a meaningful extrapolation to the thermodynamic limit can be done.

Computational complexity of the Diag-MC technique for sign-alternating series is also exponential/factorial in the diagram order and final results have to be extrapolated to the $N_{*} \rightarrow \infty$ limit. In this sense the technique does not solve the sign-problem, but offers a better route for handling it. One important difference between the configuration space volume for finite-size systems and connected Feynman diagrams is that the latter deals with the thermodynamic limit directly. Moreover, the same diagrams describe systems of different dimensions and temperature. The list of advantages does not end here because one can employ all known analytic tools to reduce the configuration space, and thus make an exponential advance towards acceptable solution of the singproblem. By simulating the self-energy instead of the Green's function (this was not done before) the configuration space is reduced to that of $G$-irreducible graphs. Using ladder diagrams we convert the standard perturbation theory in the bare potential $V$ into the series expansion in terms of $\Gamma$. Finally, the entire scheme is made self-consistent by writing diagrams in terms of exact $G$ and $Q$. Since self-consistency accounts for infinite sums of diagrams forming geometrical series the configuration space of bold-line diagrams is reduced further. All combined, the final formulation is compact enough to perform the $N_{*} \rightarrow \infty$ extrapolation reliably before error bars explode. Strictly speaking, having convergent series is not a requirement because re-summation techniques are welldefined mathematically and their work is guaranteed by theorems based on properties of analytic functions.

At the moment we do not see any obvious limitations 
of the method described here. On the contrary, we believe that it can be used to study a generic interacting many-body system, Bose or Fermi. Of course, the structure of diagrams and the optimal strategy of applying analytic tools are Hamiltonian dependent and have to be studied case-by-case. For example, in lattice models there is no urgent need to deal with the ultra-violet limit explicitly and one can proceed with the expansion in the bare interaction potential $V$; the so-called random phase approximation can be used to replace $V$ with the screened interaction potential; the latter can be combined with ladder diagrams, etc. The bold-line trick for Green's functions can be implemented in any scheme.

To summarize, we have shown that polaron type problems can be studied numerically with high accuracy using Diag-MC methods even when the corresponding dia- grammatic expansion is not sign-positive and divergent. Previously such series were regarded as hopeless numerically, to such an extent that nobody was actually trying! Using Diag-MC approach we calculated energies and effective masses of resonant Fermi-polarons in the BCS-BEC crossover region and determined that the point where the groundstate switches from the single-particle (fermionic) sector to two-particle (bosonic) sector is at $k_{F} a=1.11(2)$. This point falls inside the phase separation region for the dilute mixture of spin-down fermions in the Fermi gas of spin-up particles [19].

We are grateful to C. Lobo, S. Giorgini, and R. Combescot for valuable discussions and data exchange. The work was supported by the National Science Foundation under Grants PHY-0426881 and PHY-0653183.
[1] L. D. Landau, Phys. Z. Sowjetunion 3664 (1933).

[2] J. Appel, in Solid State Physics, eds. H. Ehrenreich, F. Seitz, and D. Turnbull, Academic, New York, Vol. 21, (1968).

[3] H. Castella and X. Zotos, Phys. Rev. B 47, 16186 (1993).

[4] H. Fröhlich, H. Pelzer, and S. Zienau, Philos. Mag. 41, 221 (1950).

[5] T. D. Schultz, Phys. Rev. 116, 526 (1959).

[6] T. D. Lee, F.E. Low, and D. Pines, Phys. Rev. 90, 297 (1953).

[7] R. P. Feynman, Phys. Rev. 97, 660 (1955); Statistical Mechanics, Reading, Benjamin (1972).

[8] J. Bonca, S. A. Trugman, and I. Batistic, Phys. Rev. B 60, 1633 (1999).

[9] H. Fehske, J. Loos, and G. Wellein, Phys. Rev. B 61, 8016 (2000).

[10] P. E. Kornilovitch, Phys. Rev. Lett. 81, 5382 (1998).

[11] C. L. Kane, P. A. Lee, and N. Read, Phys. Rev. B 39, 6880 (1989).

[12] N. V. Prokof'ev and B. V. Svistunov, arXiv:0707.4259, to appear in Phys. Rev. B.

[13] N. V. Prokof'ev and B. V. Svistunov, Phys. Rev. Lett. 81, 2514 (1998).

[14] A. S. Mishchenko, N. V. Prokof'ev, A. Sakamoto, and B. V. Svistunov, Phys. Rev. B 62, 6317 (2000).

[15] A. Bulgac and M. McNeil Forbes, Phys. Rev. A 75, 031605(R) (2007).

[16] C. Lobo, A. Recati, S. Giorgini, and S. Stringari, Phys. Rev. Lett. 97, 200403 (2006).

[17] S. Sachdev and K. Yang, Phys. Rev. B 73, 174504 (2006).

[18] J. Carlson and S. Reddy, Phys. Rev. Lett. 95, 060401 (2005).

[19] S. Pilati, S. Giorgini, arXiv:0710.1549.

[20] D. E. Sheehy and L. Radzihovsky, Phys. Rev. Lett. 96, 060401 (2006).

[21] C.-H. Pao, S.-T. Wu and S.-K. Yip, Phys. Rev. B 73, 132506 (2006).

[22] M. Iskin and C.A.R. Sa de Melo, Phys. Rev. Lett. 97, 100404 (2006).

[23] H. Hu and X.-J. Liu, Phys. Rev. A 73, 051603(R) (2006).

[24] C.-C. Chien, Q. Chen, Y. He, and K. Levin, Phys. Rev. A 74, 021602(R) (2006).
[25] M. M. Parish, F. M. Marchetti, A. Lamacraft, and B. D. Simons, Nature Phys. 3, 124 (2007); Phys. Rev. Lett. 98, (2007) 160402

[26] Y. Shin, M. W. Zwierlein, C. H. Schunck, A. Schirotzek, and W. Ketterle, Phys. Rev. Lett. 97, 030401 (2006); Y. Shin, C. H. Schunck, A. Schirotzek, W. Ketterle, condmat/0709.3027.

[27] G. B. Partridge, W. Li, R. I. Kamar, Y. A. Liao, and R. G. Hulet, Science 311, 503 (2006); G. B. Partridge, W. Li, Y. A. Liao, R. G. Hulet, M. Haque and H. T. C. Stoof, Phys. Rev. Lett. 97, 190407 (2006).

[28] P. W. Anderson, Phys. Rev. Let. 18, 1049 (1967); Phys. Rev. 164, 352 (1967).

[29] K. Yamada, A. Sakurai, M. Takeshige, Prog. Theor. Phys. 70, 73 (1983); J. Kondo, Physica B 126, 377 (1984).

[30] U. Weiss, Quantum Dissipative Systems, third edition, World Scientific, Singapore (2007).

[31] E. M. Lifshitz and L. P. Pitaevskii, Statistical Physics, Part 2, Landau and Lifshitz Course of Theoretical Physics, Volume 9, Butterworth-Heinemann, Oxford (2002).

[32] N. V. Prokof'ev and B. V. Svistunov, Phys. Rev. Lett. 99, 250201 (2007).

[33] N. V. Prokof'ev, B. V. Svistunov, and I. S. Tupitsyn, Phys. Lett. 238, 253 (1998); Sov. Phys. JETP 87, 310 (1998); M. Boninsegni, N. V. Prokof'ev, and B. V. Svistunov, Phys. Phys. Rev. Lett. 96, 070601 (2006); Rev. E 74, 036701 (2006).

[34] N. V. Prokof'ev, B. V. Svistunov, Phys. Rev. Lett. 87, 160601 (2001).

[35] G. V. Skorniakov and K. A. Ter-Martirosian, Zh. Eksp. Teor. Fiz. 31, 775 (1956) [Sov. Phys. JETP 4, 648 (1957)].

[36] M. Yu. Kagan, A. V. Klaptsov, I. V. Brodsky, R. Combescot, and X. Leyronas, Physics-Uspekhi 49, 1079 (2006).

[37] R. Combescot, A. Recati, C. Lobo, and F. Chevy, Phys. Rev. Lett. 98, 180402 (2007).

[38] D. S. Petrov, C. Salomon, and G. V. Shlyapnikov, Phys. Rev. Lett. 93, 090404 (2004).

[39] L. Viverit, C.J. Pethick, and H. Smith, Phys. Rev. A 61, 
053605 (2000).

[40] P. Pieri and G. C. Strinati, Phys. Rev. Lett. 96, 150404 (2006); E. Taylor, A. Griffin and Y. Ohashi, Phys. Rev.
A 76, 023614 (2007); M. Iskin and C.A.R. Sa de Melo, cond-mat/0709.4424. 